\title{
Suitability for coding of the Colebrook's flow friction relation expressed by symbolic regression approximations of the Wright-w function
}

\author{
Pavel Praks ${ }^{1}$ and Dejan Brkić ${ }^{1}$ \\ ${ }^{1}$ IT4Innovations, VSB - Technical University of Ostrava, Ostrava, Czechia \\ ${ }^{1}$ e-mail: pavel.praks@ vsb.cz; dejan.brkic@ vsb.cz, dejanbrkic0611@ gmail.com
}

\begin{tabular}{l} 
Article Info \\
\hline Article history: \\
Received November 2, 2020 \\
Revised December 7, 2020 \\
Accepted December 9, 2020 \\
\hline
\end{tabular}

\section{Keywords:}

Flow friction

Colebrook equation

Wright $\omega$-function

Artificial intelligence

Symbolic regression

Computational speed

\begin{abstract}
This article analyses a form of the empirical Colebrook's pipe flow friction equation given originally by the Lambert $\mathrm{W}$-function and recently also by the Wright $\omega$-function. These special functions are used to explicitly express the unknown flow friction factor of the Colebrook equation, which is in its classical formulation given implicitly. Explicit approximations of the Colebrook equation based on approximations of the Wright $\omega$-function given by an asymptotic expansion and symbolic regression were analyzed in respect of speed and accuracy. Numerical experiments on 8 million Sobol's quasiMonte points clearly show that also both approaches lead to approximately the same complexity in terms of speed of execution in computers. However, the relative error of the developed symbolic regression-based approximations is reduced significantly, in comparison with the classical basic asymptotic expansion. These numerical results indicate promising results of artificial intelligence (symbolic regression) for developing fast and accurate explicit approximations.
\end{abstract}

Copyright (C) 2020 Regional Association for Security and crisis management and European centre for operational research. All rights reserved.

\section{Corresponding Author:}

Dejan Brkić,

IT4Innovations, VSB - Technical University of Ostrava, Ostrava, Czechia.

Emails: dejan.brkic@vsb.cz, dejanbrkic0611@gmail.com

\section{Introduction}

Relation developed by Colebrook (1939) is widely used for calculation of friction factor in pipes, Eq. (1):

$\frac{1}{\sqrt{\lambda}}=-2 \cdot \log _{10}\left(\frac{2.51}{R \mathrm{e}} \cdot \frac{1}{\sqrt{\lambda}}+\frac{\varepsilon}{3.71}\right)$

In the Colebrook equation $\lambda$ is the unknown Darcy's flow friction factor, while $R e$ is the Reynolds number and $\varepsilon$ is the relative roughness of inner pipe surface (all three quantities are dimensionless). The flow friction factor $\lambda$ is locked in an implicit form through a logarithmic expression. Domains of the input parameters used in engineering practice are $4000<R e<10^{8}$ and $0<\varepsilon<0.05$, while for the output parameter $0<\lambda<$ 0.088 . The Reynolds number is an important dimensionless quantity in fluid mechanics which is used to predict transition from laminar, sheet-like flow in parallel layers to turbulent flow which is characterized with unsteady vortices, while the relative roughness $\varepsilon$ is calculated as the absolute roughness of an inner pipe surface divided by the inside diameter of a pipe, where the absolute roughness represents the average the height across the microscopic peaks and valleys above the laminar sub-layer of fluid (see Fig. 1). It is not easy to estimate actual value of absolute roughness of a pipe material (Guo et al. 2020), but it is from around $0.0025 \mathrm{~mm}$ for glass and 
plastic which are practically smooth, $0.025 \mathrm{~mm}$ new smooth concrete, around $0.15 \mathrm{~mm}$ for casted iron, $0.25 \mathrm{~mm}$ for coarse concrete, around $0.5 \mathrm{~mm}$ for rusted steel, etc. (Moody 1944).

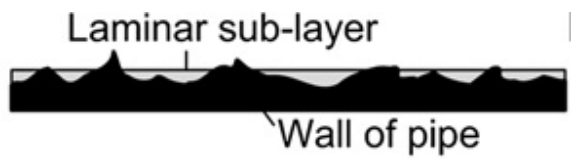

Figure 1. Absolute roughness of inner pipe surface: average height across the microscopic peaks and val-leys above the laminar sub-layer of fluid.

Depending on thickness of the laminar sub-layer which can cover partially or entirely the protrusions of pipe material, value of hydraulic roughness can varies also depending on the type of fluid but also on the Reynolds number where for the laminar flow (laminar from occurs for $R e<4000$, but can be also for the higher values of $R e$; on the other hand, turbulent flow cannot exist for $R e<4000$ ) all materials are practically smooth, where for the fully developed turbulent flow (high values of $R e$ ), laminar sub-layer does not exist and physical roughness of the surface is equal to the hydraulic roughness (Brkic 2012a).

The Colebrook equation is of empirical nature (Colebrook and White 1937). Its graphical interpretation is given by Moody diagram (Moody 1944). Number of explicit approximations of the Colebrook equation exists (Assuncao et al. 2020, Brkić 2011ab, Brkić and Ćojbašić 2017), and here we will offer few very accurate and computationally efficient which are based on the Wright $\omega$-function, a cognate of the Lambert $\mathrm{W}$-function (Corless et al. 1996). Thanks to these special functions, it is capable to transform expressions from their implicit in an explicit form, which is suitable for further processing (Brkić 2011bc, Brkić 2012c, Brkić and Praks 2019, Praks and Brkić 2020).

\section{Mathematics behind the proposed solution}

\subsection{Lambert W-function and Wright $\omega$-function}

The Lambert W-function (Corless et al. 1996, Barry et al. 2000, Fukushima 2020a) is defined as the inverse function of $f(W)=W \cdot e^{W}$, whereas the Wright $\omega$-function with $x$ as the argument solves the equation $y+$ $\ln (y)=x$. Thus, the Wright $\omega$-function (Corless and Jeffrey 2002) is a cognate of the Lambert $\mathrm{W}$-function, and here it is used to transform the Colebrook equation from the implicitly given form in respect of the unknown variable to the explicit form (Brkić and Praks 2019, Praks and Brkić 2020). Additionally, about application of the Lambert W-function to the Colebrook equation can be seen in Alfaro-Guerra et al. 2020, Vatankhah 2018, Biberg 2017, Mikata and Walczak 2016, Viccione and Tibullo 2012, etc.

However, as the further evaluation of the Wright $\omega$-function can be only approximate, approximations of the Wright $\omega$-function are developed for the various purposes and hence they are very well tested (Corless and Jeffrey 2002, Fukushima 2020b).

The Wright $\omega$-function is the Lambert $\mathrm{W}$-function with a shifted argument, and here is used for the fact that such shifted argument is not fast-growing as in the case of the Lambert W-function (Brkić 2012d). If the Lambert W-function is used for solving the Colebrook equation, an overflow error will occur in about half cases used in engineering practice (Sonnad and Goudar 2002), as the required term $W\left(e^{x}\right)$ cannot be accurately stored in registers. However, some software packages (e.g. Matlab) in such cases use a numerically stable the Wright $\omega$-function instead of the Lambert $\mathrm{W}$-function with the purpose to avoid the overflow error by the equivalence $W\left(e^{x}\right)=\omega(x)$, where e.g. for $x=500, y=W\left(e^{500}\right)=\omega(500)=493.7978$.

\subsection{Asymptotic expansion}

We will test approximations of $y=\omega(x)-x$ given by the asymptotic expansion provided by Corless and Jeffrey (2002).

The used asymptotic expansion series are given in Table 1 denoted by the symbol AE and summarized in the first four lines.

\subsection{Symbolic regression}

Symbolic regression is based on artificial intelligence and it is capable to find approximations for the certain function given by data sets. The main advantage of such approach is that the symbolic expression is found by an artificial intelligence tool and it is not prescribed in advance. The user only specifies building blocks of the 
symbolic formula (for example, arithmetic operations, logarithms, etc.). Here is used software tool Eureqa (Dubčáková 2011).

The developed symbolic regression formulas are in Table 1 denoted by the symbol SR (additional formula are in Conclusions).

\subsection{Matlab built-in implementation and WrightOmegaq library}

In Matlab, the built-in Wright $\omega$-function is denoted by the command wrightOmega. In Table 1, this Matlab built-in function is represented by the symbol "Matlab built-in". However, Table 1 also shows the results of an alternative open-source implementation of the Wright $\omega$-function, which is represented by the symbol "wrightOmegaq". The "wrightOmegaq" library works well also in GNU Octave. Table 1 presents results of two variants of the formula: exact constants and approximated constants. The "exact constants" is the exact solution given in Eq. (2), whereas the "approximated constants" is the proposed approximation suitable for engineering practice given in Eq. (3).

The aim of this comparison is to demonstrate how reducing the number of digits used in Eq. (3) will reduce the accuracy of results.

\section{Exact explicit solution of the Colebrook equation}

The Colebrook equation in term of the Wright $\omega$-function can be given as Eq. (2):

$$
\left\{\begin{array}{c}
\frac{1}{\sqrt{\lambda}}=\frac{C}{2.51} \cdot(B+y) \\
C=\frac{2 \cdot 2.51}{\ln (10)} \\
A=\frac{R e}{C} \cdot \frac{\varepsilon}{3.71} \\
B=\ln (R e)-\ln (C) \\
x=A+B \\
y=\omega(x)-x
\end{array}\right.
$$

Series about infinity of $\omega(x)-x=-\ln (x)+\sum_{l \geq 0} \sum_{m \geq 0} c_{l m} \frac{l^{m}(x)}{x^{l+m}}$ is defined in terms of Striling cycle numbers, where $c_{l m}=(-1)^{l}\left[\begin{array}{c}l+m \\ l+1\end{array}\right] / m$ !, where $\mathrm{m}$ and 1 are positive integer numbers (Rollmann and Spindler 2015). For the Colebrook equation, $\omega(x)-x$ is strictly monotonic decreasing as can be seen from Fig. 2, where $x$ for the practical engineering interest goes for the Colebrook's equation from 7 to 619 , while the diagram is plotted in Matlab as:

syms $x$

assume $(x>=7 \quad \& \quad x<=619)$

$\mathrm{f}=$ wrightOmega $(\mathrm{x})-\mathrm{x}$

fplot (f, $[7,619]$ )

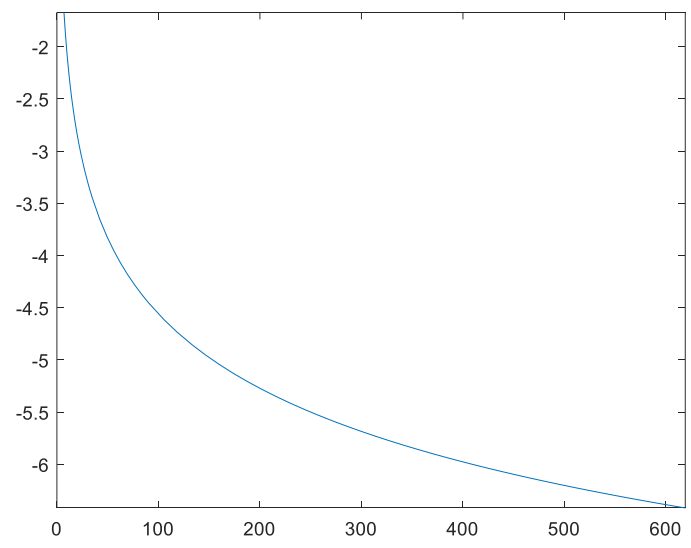

Figure 2. Strictly monotonic decreasing of $y=\omega(x)-x$ for the Colebrook equation; the horizontal axis represents $x$ and the vertical $y$ - this figure is adapted from Praks and Brkić (2020). 
One possible simplification based in the form of approximation suitable for engineering use can be, Eq. (3):

$$
\left\{\begin{array}{c}
\frac{1}{\sqrt{\lambda}} \approx 0.868589 \cdot(B+y) \\
A \approx \frac{R e \cdot \varepsilon}{8.0878} \\
B \approx \ln (R e)-0.7794 \\
x \approx A+B
\end{array}\right.
$$

Where $y=\omega(x)-x$ is approximated in Table 1 in Matlab notation. The column 'time in sec' is given for execution of the Colebrook equation for 8 million samples generated using the Sobol's Quasi Monte Carlo algorithm, which covers the entire region of interest very efficiently (Sobol et al. 1992).

Table 1. Comparison of approximations of $y=\omega(x)-x$ with related error and speed of execution; Asymptotic expansion (AE), Symbolic regression (SR).

\begin{tabular}{|c|c|c|c|c|}
\hline $\begin{array}{l}\text { Type of } \omega(x) \\
\text { approximation }\end{array}$ & Approximation of $y$ & $\begin{array}{l}\text { Relative } \\
\text { error in \% }\end{array}$ & Ratio & $\begin{array}{c}\text { Time in } \\
\text { sec }\end{array}$ \\
\hline $\mathrm{AE}$ & $y=\ln x . / x-\ln x$ & $1.52 \mathrm{E}-01$ & 1 & 0.7 \\
\hline $\mathrm{AE}$ & $y=\ln x . / x-\ln x+0.000818$ & $1.36 \mathrm{E}-01$ & 1.1 & 0.7 \\
\hline $\mathrm{AE}$ & $\mathrm{y}=\ln \mathrm{x} . / \mathrm{x}-\ln \mathrm{x}+0.5 \cdot * \ln \mathrm{x} \cdot^{*}(\ln \mathrm{x}-2) \cdot / \mathrm{x} \cdot{ }^{\wedge} 2$ & $1.18 \mathrm{E}-01$ & 1.3 & 0.9 \\
\hline $\mathrm{AE}$ & $y=\ln x . / x-\ln x+0.5 .^{*} \ln x .^{*}(\ln x-2) . / x .^{\wedge} 2-0.002$ & 9.61E-02 & 1.6 & 0.9 \\
\hline SR & $\mathrm{y}=(1.038 * \ln \mathrm{x}) . /(\mathrm{x}+0.332)-\ln \mathrm{x}$ & $5.22 \mathrm{E}-02$ & 2.9 & 0.7 \\
\hline SR & $\mathrm{y}=(1.0119 * \ln \mathrm{x}) . / \mathrm{x}-\ln \mathrm{x}+(\ln \mathrm{x}-2.3849) . / \mathrm{x} .^{\wedge} 2$ & $8.45 \mathrm{E}-03$ & 18.0 & 0.8 \\
\hline wrightOmegaq & $\mathrm{y}=$ wrightOmegaq $(\mathrm{x})-\mathrm{x}$ (approximated constants) & $2.49 \mathrm{E}-03$ & 61 & 3.8 \\
\hline wrightOmegaq & $\mathrm{y}=$ wrightOmegaq $(\mathrm{x})-\mathrm{x}$ (exact constants) & 0 & & 2.7 \\
\hline Matlab built-in & $\mathrm{y}=$ wrightOmega $(\mathrm{x})-\mathrm{x}$ & 0 & & 5158 \\
\hline
\end{tabular}

Results from Table 1 demonstrate that the speed of both used approximations (the asymptotic expansion based vs. symbolic regression based) is approximately the same, as the computation time varies from $0.7 \mathrm{sec}$ to $0.9 \mathrm{sec}$. In order to quantify the improvement of the approximations from Table 1, we computed the relative precision ratio between the basic approximation $y=\ln x . / x-\ln x$ and the given approximations. For this reason, the basic approximation $y=\ln x . / x-\ln x$ has the improvement equal to one, where the first symbolic regression approximation from Table 1 has improvement 2.9, while the second symbolic regression approximation has improvement 18 , as the relative precision is reduced from $1.52 \mathrm{E}-01 \%$ to $8.45 \mathrm{E}-03 \%$. Moreover, we can see that the symbolic regression approximations have approximately the same speed as the original asymptotic regression approximation $\mathrm{y}=\ln x . / \mathrm{x}-\ln x(0.7 \mathrm{sec}$ and $0.8 \mathrm{sec}$ vs. $0.7 \mathrm{sec})$.

Numerical experiments on 8 million Sobol's quasi-Monte points clearly show that although both approaches lead to the approximately same complexity (the computer speed is similar), the relative error of the novel symbolic regression approximation is reduced 18 times, if we compare it with the original asymptotic regression approxima-tion $y=\ln x . / x-\ln x$. We can also clearly observe from the Table 1 , that the Wright $\omega$ function built-in in Matlab is extremely slow, in comparison with the open-source library wrightOmegaq: The Wright $\omega$-function of Matlab requires 5158 seconds for the benchmark of Table 1, whereas the wrightOmegaq requires less than 4 seconds. The corresponding speed-up ratio is 5158/4 1289.

We can also see from the Table 1 that it make sense to create fast approximations of the wrightOmegaq library: The exact solution given by the wrightOmegaq library requires 2.7-3.8 seconds, whereas all the here mentioned fast approximations always require less than 1 second.

\section{Conclusions}

Numerical experiments on 8 million Sobol's quasi-Monte points clearly show that both approaches, asymptotic expansion and symbolic regression, to make approximate relations for the Colebrook equation for flow friction transformed through the Wright $\omega$-function, lead to the approximately same complexity of the obtained approximations (computer speed is also similar). However, the relative error of the two developed symbolic regression approximations is reduced by factor 3 and 18, respectively, in comparison with the classical asymptotic expansion. These numerical results indicate promising results of artificial intelligence (symbolic regression) for the area of fast and accurate explicit approximations. On the other hand, although accurate, build-in Matlab function for the Wright $\omega$-function is extremely slow. Numerical experiments in Table 1 also show that the alternative open-source implementation given by the library "wrightOmegaq" is much faster than the Matlab build-in implementation (Horchler 2017). 
As reported in Praks and Brkić (2020), as extension to the results from Table 1, approximation $\mathrm{y}=\ln x . /(\mathrm{x}-$ $0.5564 * \ln x+1.207)$. $-\ln x$, is to date the most accurate.

Acknowledgement: This work was supported by the Ministry of Education, Youth and Sports from the National Programme of Sustainability (NPS II) project "IT4Innovations excellence in science - LQ1602", from the Large Infrastructures for Research, Experimental Development, and Innovations project "e-INFRA CZ LM2018140", and by the Technology Agency of the Czech Republic through the project CEET - "Center of Energy and Environmental Technologies" TK03020027.

\section{References}

Alfaro-Guerra, M., Guerra-Rojas, R., \& Olivares-Gallardo, A. (2020). Evaluación de la profundidad de recursión de la solución analítica de la ecuación de Colebrook-White en la exactitud de la predicción del factor de fricción. Ingeniería, investigación y tecnología, 21(4), https://doi.org/10.22201/fi.25940732e.2020.21.4.036

Assuncao, G. S. C., Marcelin, D., von Hohendorff Filho, J. C., Schiozer, D. J., de Castro, M. S. (2020). Friction factor equations accuracy for single and two-phase flows. ASME 2020 39th International Conference on Ocean, Offshore and Arctic Engineering (OMAE2020), Virtual Online, August 3 - 7, 2020. Available from: https://www.researchgate.net/publication/346503366 (accessed on 06 December 2020)

Barry, D. A., Parlange, J. Y., Li, L., Prommer, H., Cunningham, C. J., \& Stagnitti, F. (2000). Analytical approximations for real values of the Lambert W-function. Mathematics and Computers in Simulation, 53(12), 95-103. https://doi.org/10.1016/S0378-4754(00)00172-5

Biberg, D. (2017). Fast and accurate approximations for the Colebrook equation. Journal of Fluids Engineering, 139(3), 031401, https://doi.org/10.1115/1.4034950

Brkić, D. (2011a). Review of explicit approximations to the Colebrook relation for flow friction. Journal of Petroleum Science and Engineering, 77(1), 34-48. https://doi.org/10.1016/j.petrol.2011.02.006

Brkić, D. (2011b). Determining friction factors in turbulent pipe flow. Chemical Engineering (New York), 119(3), 34-39. Available from: https://www.chemengonline.com/determining-friction-factors-in-turbulentpipe-flow/?printmode $=1$ (accessed on 06 December 2020)

Brkić, D. (2011b). W solutions of the CW equation for flow friction. Applied Mathematics Letters, 24(8), 13791383. https://doi.org/10.1016/j.aml.2011.03.014

Brkić, D. (2011c). An explicit approximation of Colebrook's equation for fluid flow friction factor. Petroleum Science and Technology, 29(15), 1596-1602. https://doi.org/10.1080/10916461003620453

Brkić, D. (2012a). Can pipes be actually really that smooth? International Journal of Refrigeration, 35(1), 209215. https://doi.org/10.1016/j.ijrefrig.2011.09.012

Brkić, D. (2012c). Lambert W function in hydraulic problems. Mathematica Balkanica (New Series), 26(3-4), 285-292. Available from: http://www.math.bas.bg/infres/MathBalk/MB-26/MB-26-285-292.pdf (accesed on 06 December 2020)

Brkić, D. (2012d). Comparison of the Lambert W-function based solutions to the Colebrook equation. Engineering Computations, 29(6), 617-630. https://doi.org/10.1108/02644401211246337

Brkić, D., \& Ćojbašić, Ž. (2017). Evolutionary optimization of Colebrook’s turbulent flow friction approximations. Fluids, 2(2), 15. https://doi.org/10.3390/fluids2020015

Brkić, D., \& Praks, P. (2019). Accurate and efficient explicit approximations of the Colebrook flow friction equation based on the Wright $\omega$-function. Mathematics, 7(1), 34. https://doi.org/10.3390/math7010034

Colebrook, C. F., \& White, C. M. (1937). Experiments with fluid friction in roughened pipes. Proceedings of the Royal Society of London. Series A-Mathematical and Physical Sciences, 161(906), 367-381. https://doi.org/10.1098/rspa.1937.0150

Colebrook, C.F. (1939). Turbulent flow in pipes with particular reference to the transition region between the smooth and rough pipe laws. Journal of the Institution of Civil Engineers, 11(4), 133-156. https://doi.org/10.1680/ijoti.1939.14509 
Corless, R. M., Gonnet, G. H., Hare, D. E., Jeffrey, D. J., \& Knuth, D. E. (1996). On the LambertW function. Advances in Computational Mathematics, 5(1), 329-359. https://doi.org/10.1007/BF02124750

Corless, R.M., \& Jeffrey, D.J. (2002). Wright $\omega$ Function. In: Calmet, J., Benhamou, B., Caprotti, O., Henocque, L., \& Sorge, V. (eds.) Artificial Intelligence, Automated Reasoning, and Symbolic Computation; AISC 2002, Calculemus 2002, Lecture Notes in Computer Science, vol. 2385, pp. 76-89. Berlin/Springer, Heidelberg. https://doi.org/10.1007/3-540-45470-5 10

Dubčáková, R. (2011). Eureqa: software review. Genetic Programming and Evolvable Machines, 12(2), 173178. https://doi.org/10.1007/s10710-010-9124-Z

Fukushima, T. (2020a). Precise and fast computation of Lambert $\mathrm{W}$ function by piecewise minimax rational function approximation with variable transformation. Available online: https://doi.org/10.13140/RG.2.2.30264.37128 (accessed on 05 December 2020)

Fukushima, T. (2020b). Fast computation of Wright $\omega$ function by piecewise minimax rational function approximation. Available online: https://doi.org/10.13140/RG.2.2.27086.28481 (accessed on 06 December 2020)

Guo, X., Wang, T., Yang, K., Fu, H., Guo, Y., \& Li, J. (2020). Estimation of equivalent sand-grain roughness for coated water supply pipes. Journal of Pipeline Systems Engineering and Practice 11(1), 04019054. https://doi.org/10.1061/(ASCE)PS.1949-1204.0000433

Horchler, A.D. (2017). Complex double-precision evaluation of the Wright Omega function. Available online: https://github.com/horchler/wrightOmegaq (accessed on 28 December 2019)

Mikata, Y., \& Walczak, W.S. (2016). Exact analytical solutions of the Colebrook-White equation. Journal of Hydraulic Engineering, 142(2), 04015050. https://doi.org/10.1061/(ASCE)HY.1943-7900.0001074

Moody, L.F. (1944) Friction factors for pipe flow. Transactions of the American Society of Mechanical Engineers, 66(8), 671-684.

Praks, P., \& Brkić, D. (2020). Review of new flow friction equations: Constructing Colebrook explicit correlations accurately. Revista Internacional de Métodos Numéricos para Cálculo y Diseño en Ingeniería, 36(3), 41, https://doi.org/10.23967/j.rimni.2020.09.001

Rollmann, P., \& Spindler, K. (2015). Explicit representation of the implicit Colebrook-White equation. Case Studies in Thermal Engineering, 5, 41-47. https://doi.org/10.1016/j.csite.2014.12.001

Sobol, I. M., Turchaninov, V. I., Levitan, Y. L., \& Shukhman, B. V. (1992). Quasi-random sequence generators; Distributed by OECD/NEA Data Bank; Keldysh Institute of Applied Mathematics; Russian Academy of Sciences: Moscow, Russia. 1992. https://ec.europa.eu/jrc/sites/jrcsh/files/LPTAU51.rar (accessed on 28 December 2019)

Sonnad, J. R., \& Goudar, C. T. (2004). Constraints for using Lambert W function-based explicit ColebrookWhite equation. Journal of Hydraulic Engineering, 130(9), 929-931. https://doi.org/10.1061/(ASCE)0733$\underline{\text { 9429(2004)130:9(929) }}$

Vatankhah, A.R. (2018). Approximate analytical solutions for the Colebrook equation. Journal of Hydraulic Engineering, 144(5), 06018007. https://doi.org/10.1061/(ASCE)HY.1943-7900.0001454

Viccione, G., \& Tibullo, V. (2012). An effective approach for designing circular pipes with the ColebrookWhite formula. AIP Conference Proceedings, 1479, 205-208. https://doi.org/10.1063/1.4756098 